\title{
A snapshot on radiotherapy for head and neck cancer patients during the COVID-19 pandemic: a survey of the Italian Association of Radiotherapy and Clinical Oncology (AIRO) head and neck working group
}

\author{
Francesca De Felice ${ }^{1}$ - Elisa D'Angelo ${ }^{2} \cdot$ Rossana Ingargiola $^{3} \cdot$ Nicola Alessandro lacovelli $^{3}$. Daniela Alterio ${ }^{4}$. \\ Pierfrancesco Franco ${ }^{5}$. Pierluigi Bonomo ${ }^{6}$. Anna Merlotti ${ }^{7}$ - Almalina Bacigalupo ${ }^{8}$. Marta Maddalo ${ }^{9}$. \\ Alessia Di Rito ${ }^{10}$. Giuseppe Fanetti ${ }^{11}$. Ida D'Onofrio ${ }^{12}$. Francesco Dionisi ${ }^{13}$. Francesco Miccichè ${ }^{14}$. \\ Marianna Trignani ${ }^{15}$. Daniela Musio ${ }^{1}$. Fabiola Paiar ${ }^{16}$. Domenico Attilio Romanello ${ }^{17,18} \cdot$ Vittorio Donato $^{19}$. \\ Ester Orlandi ${ }^{18}{ }_{\mathbb{C}}$
}

Received: 15 July 2020 / Accepted: 21 September 2020 / Published online: 6 October 2020

(C) Italian Society of Medical Radiology 2020

\begin{abstract}
Objectives The objective of the paper was to assess real-life experience in the management of head and neck cancer (HNC) patients during the COVID-19 pandemic in radiotherapy departments and to evaluate the variability in terms of adherence to American Society of Radiation Oncology (ASTRO) and European Society for Radiotherapy and Oncology (ESTRO) recommendations.

Materials and methods In May 2020, an anonymous 30-question online survey, comparing acute phase of outbreak and preCOVID-19 period, was conducted. Two sections exploited changes in general management of HNC patients and different HNC primary tumors, addressing specific statements from ASTRO ESTRO consensus statement as well.

Results Eighty-eight questionnaires were included in the demographic/clinical workflow analysis, and 64 were analyzed for treatment management. Forty-eight percent of radiotherapy departments became part of oncologic hubs. First consultations reduced, and patients were addressed to other centers in 33.8 and $18.3 \%$ of cases, respectively. Telematic consultations were used in 50\% of follow-up visits and $73.9 \%$ of multidisciplinary tumor board discussions. There were no practical changes in the management of patients affected by different primitive HNCs. Hypofractionation was not favored over conventional schedules.

Conclusions Compared to pre-COVID era, the clinical workflow was highly re-organized, whereas there were no consistent changes in RT indications and schedules.
\end{abstract}

Keywords COVID-19 $\cdot$ Head and neck cancer $\cdot$ Radiotherapy $\cdot$ Survey

Francesca De Felice and Elisa D'Angelo equally contributed to the current work.

Vittorio Donato: President of AIRO (Italian Association of Radiotherapy and Clinical Oncology), Italy.

Electronic supplementary material The online version of this article (https://doi.org/10.1007/s11547-020-01296-7) contains supplementary material, which is available to authorized users.

Ester Orlandi

ester.orlandi@cnao.it

Extended author information available on the last page of the article

\section{Introduction}

One of the basic principles of the Italian association of radiotherapy and clinical oncology (AIRO) head and neck working group is to actively support and disseminate evidencebased and utmost quality clinical practice [1]. The current coronavirus disease 2019 (COVID-19) pandemic has caused two urgent needs: i) to consistently revise the healthcare system organization, including transformation of specific centers into regional referral hubs for cancer patients; ii) to provide adequate safety recommendations to continue ensuring high-quality cancer treatments. The management of head 
and neck cancer (HNC) patients represents a unique challenge, mainly due to patients' higher risk for severe acute respiratory syndrome coronavirus 2 (SARS-CoV-2) infection as a result of their vulnerable general clinical situation, including the presence of underlying conditions, immunosuppressive status, older age [2,3]. Although a joint treatment consensus statement has been published by both the American Society of Radiation Oncology (ASTRO) and the European Society for Radiotherapy and Oncology (ESTRO), Italy has been involved in this pandemic circumstance from the very beginning, and the experience of Italian HNC staff could be useful to maintain high standards in HNC care [4].

In this context, we assessed the situation Italian radiation oncologists had to deal with in the last 3 months to gain information about real-life experience in the management of HNC patients during the COVID-19 pandemic. The variability in terms of adherence to ASTRO ESTRO recommendations was also assessed.

\section{Methods and materials}

A 30-question online survey was conducted using the SurveyMonkey platform [5]. Questions were set up in two main sections: (1) how the general management of HNC patients and hospital organization were changed in the acute phase of the outbreak (March 2020-May 2020) compared to the pre-COVID-19 period (October 2019-December 2019) and (2) how the management of different HNC primary tumors, including laryngeal, hypopharyngeal, oropharyngeal and oral cavity squamous cell carcinoma, salivary gland tumor, nasopharyngeal carcinoma and nasal/paranasal sinus cancer was changed. In particular, specific statements from the ASTRO ESTRO consensus statement were addressed. Questions concerning induction chemotherapy and clinical treatment approach in both metastatic and oligometastatic disease at diagnosis and in elderly patients were also explored. Details are listed in the supplementary materials. Demographic information was collected at the beginning of the survey. Multiple-choice answers were allowed, with certain responses prompting additional clarifying items. The Survey was initially developed by a core group, with critical review and revision from all coauthors. The final version was approved by the AIRO scientific committee and distributed via e-mail to the entire AIRO membership list. The first communication was sent to members on May 19, 2020, with a follow-up e-mail sent to non-responders on May 22, 2020 before closing the survey on May 24, 2020. Responses were recorded anonymously and only fully completed questionnaires were included in the final analysis. Standard descriptive statistics were used to evaluate the distribution of each response. Data were reported as means or percentages.

\section{Results}

In total, $89 \mathrm{HNC}$ specialists responded. Questionnaires with missing demographic data $(n=1)$ or missing essential information regarding treatment strategies $(n=24)$ were excluded. Therefore, 88 questionnaires were included in the demographic/clinical workflow analysis and 64 questionnaires were analyzed in the treatment management analysis.

\section{Demographic information}

Geographical distribution of responders across Italy is depicted in Fig. 1. Most of radiation oncologists (61.4\%) worked in public university hospital and are used to treat from 50 to $100 \mathrm{HNC}$ patients annually. Detailed demographic characteristics are listed in Table 1.

\section{Clinical workflow}

In response to COVID-19 emergency, $48.8 \%$ of radiotherapy departments were part of a center that began operating as a hub for oncologic patients. Compared to the pre-COVID-19 period (October 2019-December 2019), the number of first outpatient consultation for HNC patients remained stable in $60 \%$ of departments, while a reduction was recorded in $33.8 \%$ of cases. Departments reduced their activity by

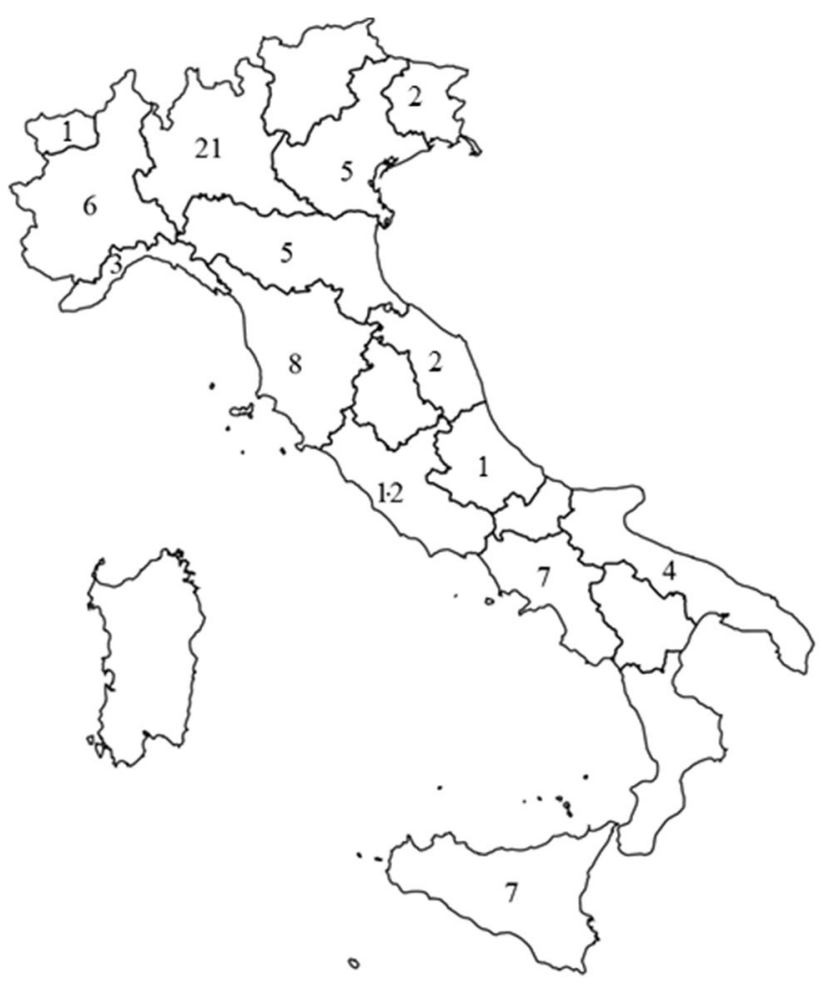

Fig. 1 Geographical distribution of responders across Italy 
Table 1 Multiple choice possible

Technique of $R T$

$\begin{array}{ll}\text { IMRT } & 60.23 \% \\ \text { VMAT } & 85.23 \% \\ \text { Hadrontherapy } & 4.55 \% \\ \text { Brachytherapy } & 7.95 \% \\ \text { 3D-CRT } & 35.23 \%\end{array}$

Number of treated patients per year

\begin{tabular}{ll}
$<50$ & $29.76 \%$ \\
$50-100$ & $46.43 \%$ \\
$>100-150$ & $16.67 \%$ \\
$150-200$ & $2.38 \%$ \\
$>200$ & $4.76 \%$ \\
\hline
\end{tabular}

less than $5 \%, 5-10 \%$ and up to $20 \%$ in $26 \%, 37 \%$ and $37 \%$ of cases, respectively. Only $2.5 \%$ of specialists declared an increase in the amount of first consultations. In $3.7 \%$ of cases, HNC patients independently postponed the first evaluation. Despite acute phase of COVID-19, most patients (up to 20 per department; $39.7 \%$ departments) came from a different region. Due to COVID-19 pandemic, $18.3 \%$ of responders referred patients to other centers for treatment. Concerning follow-up visits, $50 \%$ of participants used telematic consultations. In $16.7 \%$ of cases follow-up visits were canceled, whereas $30.8 \%$ of departments observed a reduction in follow-up visits and the remainder $2.5 \%$ were unchanged. Compared to the pre-COVID-19 period (October 2019-December 2019), the reduction rate was higher than $20 \%$ in $62.5 \%$ of cases. Most of centers $(53.9 \%)$ confirmed diagnostic imaging examinations, during follow-up, in case of symptoms or clinical suspicion of recurrence (urgent follow-up). The others responders postponed $(20.5 \%)$ or prescribe examinations as usual $(25.6 \%)$, whenever possible. Telematic multidisciplinary tumor board discussion was assured in $73.9 \%$ of departments, with several limitations in $33 \%$ of cases mainly due to concurrent activities related to COVID-19 management.

\section{Treatment management in head and neck cancer}

In case of COVID-19 positive HNC patients (20.9\%), all specialists postponed the beginning of radiotherapy after negativization of swab test. In total, $15.6 \%$ of departments experienced cases of intercurrent COVID-19 infection during treatment and in a minority of cases $(2.9 \%)$ they favored strategies avoiding treatment interruption. The majority of these cases $(72.7 \%)$ were recorded in northern Italy. Radiation therapy was interrupted until the infection resolved (repeat negative swab test) in $80 \%$ of cases, whereas the remainder $(20 \%)$ continued therapy. There were no practical changes in the management of patients affected with oropharyngeal carcinoma (both HPV-positive and HPVnegative), nasopharyngeal carcinoma, nasal/paranasal sinus cancer, oral cavity with positive/close margins and laryngeal carcinoma (T3-T4). In particular, no delays or omissions were reported in both definitive and postoperative settings. Regarding salivary glands cancer, $15.7 \%$ of specialist delayed adjuvant treatment in low-grade carcinomas, whereas there were no differences in high-grade tumor management compared to standard indications. Induction chemotherapy to retain organ functions and tumor shrinkage in cases of large tumors was considered in selected cases by $29.7 \%$ departments. In total, radiation oncologists favored the hypo-fractionated scheme in T1 and T2 glottic cancer $(15.7 \%)$, oligometastatic disease at diagnosis $(25 \%)$ and elderly patients (14.1\%). In the palliative setting, most of department $(78.1 \%$ ) reported no changes in patient management; single fraction was prescribed in $15.7 \%$ of cases. No consistent differences in HNC treatment across Italian regions was reported.

\section{Discussion}

This survey represents an attempt to comprehensively assess the national management of HNC patients during the early stages of COVID-19 pandemic in a country such as Italy where the healthcare system was largely overwhelmed by the epidemic. Most radiation therapy departments were affected by the COVID-19 outbreak, and HNC clinical workflow and contingency measures were adapted very early to the new reality. First visit and urgent follow-up visits were prioritized. The volume of routine follow-up clinic consultations decreased, and ambulatory was accordingly assured online. The vast majority of institutions continued HNC multidisciplinary team meetings using Web platforms to define the appropriate patient-centered treatment strategy. Overall, intensity-modulated radiotherapy (IMRT) technique was the standard maintained, and hypo-fractionated regimen was not favored over conventional schedules. Surgery maintained the prominent role in the management of patients with oral cavity carcinoma, salivary gland tumor and nasal/paranasal sinus cancer. In these clinical scenarios, a more prolonged delay in adjuvant RT for low-grade salivary gland cancers was considered to optimize resources without significant change in the chance of cure. Although efforts were displayed to guarantee timely adjuvant RT, we might expect a potential remarkable increased treatment package time due to a reduction of surgical oncology activity, with a possible detrimental HNC patients' outcome [6]. Interestingly, despite the rapid spread of COVID-19 has placed tremendous strain on the Italian healthcare system, the vast majority of radiotherapy departments $(79.1 \%)$ had no experience of COVID-19 positive HNC. While there was a unanimous 
agreement to defer RT treatment in COVID-19 positive patients, a minority of radiation oncologists (2.9\%) favored strategies avoiding treatment interruption in cases who became COVID-19 positive during treatment. Although an heterogeneity in terms of daily practice attitude of radiation oncologists operating in different Italian regions with different COVID-19 spread rates toward HNC management was perceptible, the present survey was not designed to demonstrate any significant difference among the single Italian institutions, but rather to describe the real management in the acute phase for further investigation. Overall, we can assume that strictly measures to reduce exposures among HNC patients and care teams were adopted to maintain clinical assistance and achieve optimal health outcomes for patients. Italian radiation oncologists remain grounded in evidence and best practice. Accordingly, i) hypofractionated approach was implemented for early laryngeal cancer (T1-2) cases and in metastatic and oligometastatic setting; ii) induction chemotherapy as well as concomitant chemotherapy in elderly patients did not have a primary role in the routine HNC recommendations. From Italian experience thus far, pre-COVID-19 standard RT treatment strategy should be preserved.

The provision of $\mathrm{HNC}$ radiation departments in Italy during the acute phase of the COVID-19 pandemic seems to be not fully in line with ASTRO and ESTRO practice recommendations. This could be secondary to several reasons. Italy was first European Nation involved in COVID-19 pandemic $[7,8]$. The outbreak impacted dramatically on the healthcare systems including RT Departments, with sudden uncertainties on the possibility to apply existing RT protocols and procedures and the need to implement new ones. Anyways, International guidelines were not yet available; thus, this may have had an impact on the data emerged from the survey. The ASTRO and ESTRO consensus reported do not interrupt RT (except for palliation, where a single fraction could be used) in all COVID 19 positive patients [3]. In the scenario of emergency COVID-19 phase, the more pursed Italian strategy was to interrupt treatment regardless of intent of RT in patients showing severe (suspected) symptoms. Finally, we observed a weak push toward the adoption of hypofractionated schemes. We believe that the substantial uncertainty regarding the ASTRO and ESTRO consensus statement is the absence of strong evidence-based scientific support favoring hypo-fractionated data, as authors declared [3]. No evidence-based indications may exacerbate treatment outcomes up to a point of irreversibility and the collective benefits of treatment failure may easily outweigh the collective risks of reduced treatment time and overall access to radiotherapy. In any cases, we have to specifically target during follow-up those patients treated with hypofractionated regimens for specific cancer types and settings, in order to detect early recurrence disease. A control system should assess the effectiveness of the rescheduling follow-up visits or the use of phone, in terms of timely evaluating potential recurrences suitable for salvage therapies and reduction of time consuming (both for patients and health workers).

This study has several limitations. While Italy has 195 radiotherapy centers, only 88 respondents participated in this survey. But the questionnaire response was strictly limited to one respondent per institution to reduce overlap bias. In addition, taking into account the largely different impact of COVID-19 along the north-south direction, the geographical distribution of participating centers in the survey may have somehow biased the interpretation of our results. In summary, the information presented here could serve as a practical method to help guiding strategies in $\mathrm{HNC}$ population during this critical circumstance.

\section{Conclusions}

COVID-19 impacted on HNC radiotherapy practice in Italy as a whole. Compared to pre-COVID era, the clinical workflow was highly re-organized, whereas there were no consistent changes in RT indications and schedules. For sure, the impact of COVID-19 on HNC management should continue to be closely monitored.

Acknowledgements The Authors thank the Scientific Committee and the Board of the AIRO for the critical revision and approval of the paper.

\section{Compliance with ethical standards}

Conflict of interest The authors declare that they have no conflict of interest.

Ethics approval Research did not involve human participant and/or animals.

Consent to participate/Consent to publish Informed consent: not applicable.

\section{References}

1. https://www.radioterapiaitalia.it

2. Chen N, Zhou M, Dong X, Qu J, Gong F, Han Y, Qiu Y, Wang J, Liu Y, Wei Y, Xia J, Yu T, Zhang X, Zhang L (2020) Epidemiological and clinical characteristics of 99 cases of 2019 novel coronavirus pneumonia in wuhan, China: a descriptive study. Lancet 395(10223):507-513

3. Yan F, Nguyen SA. Head and neck cancer: a HIGH-RISK population for COVID-19. Head Neck. Published online April 27, 2020 : hed.26209. https://doi.org/10.1002/hed.26209

4. Thomson DJ, Palma D, Guckenberger M, Balermpas P, Beitler JJ, Blanchard P, Brizel D, Budach W, Caudell J, Corry J, Corvo R, Evans M, Garden AS, Giralt J, Gregoire V, Harari PM, Harrington K, Hitchcock YJ, Johansen J, Kaanders J, Koyfman S, Langendijk 
JA, Le Q, Lee N, Margalit D, Mierzwa M, Sandro Porceddu S, Soong YL, Sun Y, Thariat J, Waldron J, Yom SS (2020) Practice recommendations for risk-adapted head and neck cancer radiation therapy during the COVID-19 pandemic: an ASTRO-ESTRO consensus statement [published online ahead of print, $2020 \mathrm{Apr}$ 14]. Int J Radiat Oncol Biol Phys S0360-3016(20)31034-8

5. https://www.surveymonkey.com

6. Werner MT, Carey RM, Albergotti WG, Lukens JN, Brody RM (2020) Impact of the COVID-19 pandemic on the management of head and neck malignancies. Otolaryngol Head Neck Surg 162(6):816-817. https://doi.org/10.1177/0194599820921413
7. Anzolin E, Amante A (2020) Coronavirus outbreak grows in northern Italy, 16 cases reported in one day. Avvenire

8. Reuters T (2020) Coronavirus. Colpite tutte le regioni. La Protezione civile: ecco i numeri aggiornati”. Avvenire

Publisher's Note Springer Nature remains neutral with regard to jurisdictional claims in published maps and institutional affiliations.

\section{Affiliations}

Francesca De Felice ${ }^{1}$ Elisa D'Angelo ${ }^{2} \cdot$ Rossana Ingargiola $^{3} \cdot$ Nicola Alessandro lacovelli $^{3} \cdot$ Daniela Alterio $^{4}$. Pierfrancesco Franco ${ }^{5}$. Pierluigi Bonomo ${ }^{6}$. Anna Merlotti ${ }^{7}$. Almalina Bacigalupo ${ }^{8} \cdot$ Marta Maddalo $^{9}$. Alessia Di Rito ${ }^{10}$. Giuseppe Fanetti ${ }^{11} \cdot$ Ida D$^{\prime}$ Onofrio ${ }^{12}$. Francesco Dionisi $^{13}$. Francesco Miccichè ${ }^{14}$. Marianna Trignani ${ }^{15}$. Daniela Musio ${ }^{1} \cdot$ Fabiola Paiar $^{16} \cdot$ Domenico Attilio Romanello $^{17,18} \cdot$ Vittorio Donato $^{19}$. Ester Orlandi ${ }^{18}$

1 Department of Radiotherapy, Policlinico Umberto I, "Sapienza" University of Rome, Rome, Italy

2 Radiation Oncology, University Hospital, AOU of Modena, Modena, Italy

3 Radiation Oncology Unit 2, Fondazione IRCCS Istituto Nazionale dei Tumori di Milano, Milan, Italy

4 Division of Radiation Oncology, IEO, European Institute of Oncology IRCCS, Milan, Italy

5 Radiation Oncology, Department of Oncology, University of Turin School of Medicine, Turin, Italy

6 Radiation Oncology, Azienda Ospedaliero-Universitaria Careggi, Florence, Italy

7 Department of Radiation Oncology, S. Croce and Carle Teaching Hospital, Cuneo, Italy

8 Radiation Oncology, Policlinico San Martino IRCCS, Genoa, Italy

9 Department of Radiation Oncology, ASST Spedali Civili of Brescia, Brescia, Italy

10 Radiation Oncology Unit, IRCCS Istituto Tumori "Giovanni Paolo II", Bari, Italy
11 Division of Radiation Oncology, Centro di Riferimento Oncologico di Aviano (CRO) IRCCS, Aviano, Italy

12 Unit of Radiation Oncology, Ospedale del Mare, Naples, Italy

13 Proton Therapy Unit, Department of Oncology, Azienda Provinciale per i Servizi Sanitari, APSS, 38123 Trento, Italy

14 Radiation Oncology, Fondazione Policlinico Universitario Agostino Gemelli IRCCS, Rome, Italy

15 Department of Radiation Oncology, "SS. Annunziata" Hospital, "G. D’Annunzio" University of Chieti, Chieti, Italy

16 Radiation Oncology Unit S, Chiara University Hospital of Pisa, Pisa, Italy

17 School of Medicine, University of Milan-Bicocca, Milan, Italy

18 National Center of Oncological Hadrontherapy (Fondazione CNAO), Strada Campeggi, 53, 27100 Pavia, Italy

19 Radiation Oncology Division, Oncology and Specialty Medicine Department, San Camillo-Forlanini Hospital, Rome, Italy 